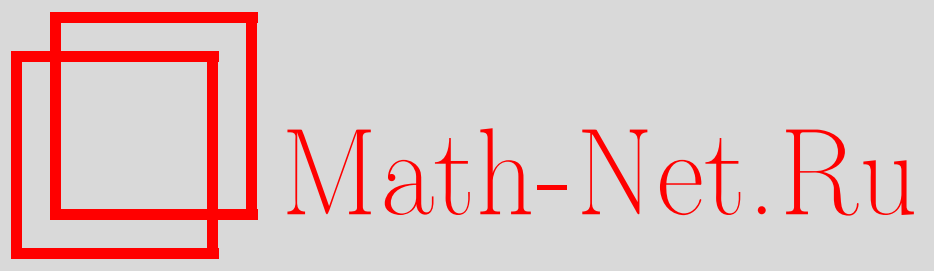

В. Р. Фаталов, Точные асимптотики типа Лапласа для гауссовской меры Боголюбова: многообразие точек минимума функционала действия, ТМФ, 2017, том 191, номер 3, 456-472

DOI: https://doi.org/10.4213/tmf9171

Использование Общероссийского математического портала Math-Net.Ru подразумевает, что вы прочитали и согласны с пользовательским соглашением http://www . mathnet.ru/rus/agreement

Параметры загрузки:

IP : 35.173 .219 .149

26 апреля 2023 г., 18:32:33

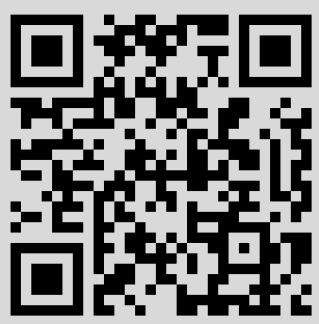




\title{
ФИЗИКА
}

Том 191, № 3

июнь, 2017

(C) 2017 г.

В. Р. Фаталов*

\section{ТОЧНЫЕ АСИМПТОТИКИ ТИПА ЛАПЛАСА ДЛЯ ГАУССОВСКОЙ МЕРЫ БОГОЛЮБОВА: МНОГООБРАЗИЕ ТОЧЕК МИНИМУМА ФУНКЦИОНАЛА ДЕЙСТВИЯ}

\begin{abstract}
Доказана теорема о точной асимптотике больших уклонений меры Боголюбова в $L^{p}$-норме для $p=4,6,8,10$ при $p>p_{0}$, где $p_{0}=2+4 \pi^{2} / \beta^{2} \omega^{2}-$ пороговое значение, $\beta>0$ - обратная температура, $\omega>0$ - собственная частота гармонического осциллятора. Исследование проводится методом Лапласа в функциональных пространствах для гауссовских мер.
\end{abstract}

Ключевые слова: мера Боголюбова, метод Лапласа в банаховом пространстве, функционал действия, многообразие точек минимума.

DOI: https://doi.org/10.4213/tmf9171

\section{1. ВВЕДЕНИЕ И ФОРМУЛИРОВКА ОСНОВНЫХ РЕЗУЛЬТАТОВ}

В статьях [1]-[3] была введена и изучена гауссовская мера Боголюбова, имеющая важное значение в теории статистического равновесия квантовых систем [4]. Гауссовский процесс Боголюбова - это стационарный гауссовский процесс $\xi(t), t \in[0, \beta]$, с нулевым средним и ковариационной функцией

$$
B(t-s) \equiv B(t, s):=\mathbf{E} \xi(t) \xi(s)=\frac{1}{2 m \omega \operatorname{sh}(\beta \omega / 2)} \operatorname{ch}\left(\omega|t-s|-\frac{\beta \omega}{2}\right) .
$$

Здесь и ниже $\mathbf{E}$ - оператор математического ожидания, взятый относительно вероятности $\mathbf{P}, m>0$ - масса частицы, $\beta>0$ - обратная температура, $\omega>0$ собственная частота гармонического осциллятора [2].

Согласно одному общему результату из теории гауссовских процессов (см. монографию [5], § 9.2) процесс Боголюбова $\xi(t)$ имеет п. н. непрерывные траектории на отрезке $[0, \beta]$. Используя формулу (1.1), находим, что $\mathbf{E}[\xi(\beta)-\xi(0)]^{2}=0$, следовательно, $\xi(0)=\xi(\beta)$ п. н. Учитывая вышеизложенное, заключаем, что траектории

Работа выполнена при поддержке РФФИ (грант № 11-01-00050).

*Механико-математический факультет, Московский государственный университет им. М. В. Ломоносова, Москва, Россия. E-mail: vrfatalov@yandex.ru 
гауссовского процесса Боголюбова принадлежат п. н. пространству $C^{0}[0, \beta]$ непрерывных на отрезке $[0, \beta]$ функций $x(t)$ с равномерной метрикой, удовлетворяющих условию $x(0)=x(\beta)$. Распределение $\mu_{\mathrm{B}}$ процесса $\xi(t)$ в пространстве $C^{0}[0, \beta]$ и называется гауссовской мерой Боголюбова.

В статье [2] были доказаны квадратурные формулы для приближенного вычисления некоторых функциональных интегралов по мере Боголюбова. В дополнение к результатам работы [2] в статье [6] на основе метода Лапласа были получены приближенные асимптотические формулы для одного класса функциональных интегралов, взятых по мере Боголюбова. В качестве основного функционала в работе [6] была взята $L^{p}$-норма при $p>0$. В результате исследований асимптотических свойств меры Боголюбова в работе [6] было установлено существование порогового значения

$$
p_{0} \equiv p_{0}(\beta \omega):=2+\frac{4 \pi^{2}}{\beta^{2} \omega^{2}} .
$$

Оказалось, что вычисление асимптотик интегралов типа Лапласа и вероятностей больших уклонений для меры Боголюбова в случае $L^{p}$-функционалов резко усложняется при $p>p_{0}$. Это связано с тем, что при $0<p<p_{0}$ соответствующая экстремальная задача для функционала действия в методе Лапласа имеет всего два решения, в то время как при $p>p_{0}$ указанная задача имеет многообразие решений. Отметим, что существование того же порогового значения $p_{0}$ установлено в статье [7] при исследовании рядов теории возмущения в квантовой механике.

В настоящей работе мы доказываем теорему о точной асимптотике больших уклонений меры Боголюбова в $L^{p}$-норме при $p>p_{0}$ для значений $p=4,6,8,10$. Метод доказательства полученных в статье результатов - это метод Лапласа в функциональных пространствах для гауссовских мер, изложенный в статье [8].

Многие утверждения ниже мы формулируем в достаточно общем виде, рассматривая случай $p>2$, это сделано с целью возможного обобщения основного результата статьи - теоремы 1 - на другие значения параметра $p$.

Перейдем к точной формулировке полученных результатов. Сформулированные ниже определения и построения аналогичны изложенным в статье [6], но содержат существенные отличия.

Для фиксированного числа $2<p<\infty$ введем следующие функции:

$$
\begin{array}{cl}
J(\delta) \equiv J_{p}(\delta):=\int_{\delta}^{1} \frac{d t}{\sqrt{\frac{1-\delta^{2}}{1-\delta^{p}}\left(1-t^{p}\right)-\left(1-t^{2}\right)}}, \\
M(\delta) \equiv M_{p}(\delta):=\int_{\delta}^{1} \frac{t^{p} d t}{\sqrt{\frac{1-\delta^{2}}{1-\delta^{p}}\left(1-t^{p}\right)-\left(1-t^{2}\right)}}, \quad 0<\delta<1 .
\end{array}
$$

Из определений получаем неравенства

$$
0<\delta^{p} J(\delta)<M(\delta)<J(\delta), \quad 0<\delta<1 .
$$

Несложно убедиться, что функции $J(\delta)$ и $M(\delta)$ корректно определены и непрерывны; некоторые свойства функции $J(\delta)$ изложены в статье [6]. Здесь мы отметим, что подынтегральные функции в выражениях (1.2), (1.3) имеют интегрируемые особенности при $t=\delta$ и $t=1$. В лемме 5 из работы [6] также доказаны следующие 
соотношения, справедливые при $p>2$ :

$$
\lim _{\delta \downarrow 0} J_{p}(\delta)=+\infty, \quad \lim _{\delta \uparrow 1} J_{p}(\delta)=\frac{\pi}{\sqrt{p-2}} .
$$

Доопределим по непрерывности

$$
J_{p}(1):=\lim _{\delta \uparrow 1} J_{p}(\delta)=\frac{\pi}{\sqrt{p-2}} .
$$

Положим

$$
\mathcal{P}=\left\{p=4,6,8,10 \text { и при этом } p>p_{0}\right\} .
$$

Проводя дальнейшие исследования асимптотических свойств меры Боголюбова, автор обнаружил, что доказательство леммы 1 из работы [6] содержит неточность. В настоящей работе дано новое строгое доказательство указанной леммы для значений $p \in \mathcal{P}$. Видоизмененная формулировка леммы 1 из [6] имеет следующий вид.

Лемма 1. 1. Пусть фиксировано $p>2+4 \pi^{2} / \beta^{2} \omega^{2}$. Тогда уравнение

$$
J_{p}(\delta)=\frac{\beta \omega}{2}, \quad 0<\delta<1,
$$

имеет хотя бы один коренъ.

2. Для значений $p \in \mathcal{P}$ уравнение (1.7) имеет единственный корень, который мы обозначим через $\delta_{0} \equiv \delta_{0}(p, \beta \omega)$.

Доказательство леммы 1 дано ниже в разделе 2 .

ЗАмечАниЕ 1. Отметим, что все результаты статьи [6] остаются в силе, кроме логарифмических соотношений (1.18), (1.25), (1.43). Отметим также, что для множеств $\mathcal{M}_{0}, \mathcal{M}_{1}$, определенных в формуле (5.12) из [6], выполнено равенство $\mathcal{M}_{0}=\mathcal{M}_{1}$, поскольку функция $x_{1}$ из (5.11) в [6] принадлежит $\mathcal{M}_{0}$. Таким образом, для значений $p \in \mathcal{P}$ минимизирующее многообразие $\mathcal{M}$ из леммы 10 в [6] имеет более простой вид: $\mathcal{M}=\mathcal{M}_{0} \cup\left[-\mathcal{M}_{0}\right]$ (см. также лемму 5 в разделе 4 настоящей работы).

В силу леммы 4 из [6] имеют место неравенства

$$
\delta_{0}(4, \beta \omega)>\delta_{0}(6, \beta \omega)>\delta_{0}(8, \beta \omega)>\delta_{0}(10, \beta \omega) .
$$

Для $p \in \mathcal{P}$ положим

$$
b_{0}=b_{0}(p, \beta \omega):=\left(\frac{\beta \omega}{2 M\left(\delta_{0}\right)}\right)^{1 / p}, \quad h_{0}:=b_{0} \delta_{0}, \quad \delta_{\infty}:=\frac{1}{\operatorname{ch}(\beta \omega / 2)} .
$$

В силу леммы 12 из [6] для фиксированных $p \in \mathcal{P}, \beta>0, \omega>0$ справедливы неравенства $0<\delta_{\infty}<\delta_{0}<h_{0}<1<b_{0}$. Определим также важный параметр (множитель Лагранжа)

$$
\lambda_{0}=\lambda_{0}(p, \beta \omega):=\beta^{2} \omega^{2} \frac{b_{0}^{2}-h_{0}^{2}}{2\left(b_{0}^{p}-h_{0}^{p}\right)}=\beta^{2} \omega^{2} \frac{1-\delta_{0}^{2}}{2\left(1-\delta_{0}^{p}\right) b_{0}^{p-2}}>0 .
$$

Для $p \in \mathcal{P}$ справедливы следующие полезные оценки (см. сооношение (1.11) в [6]):

$$
2 \beta \omega \operatorname{th} \frac{\beta \omega}{2} \leqslant p \lambda_{0} \leqslant \beta^{2} \omega^{2} .
$$


Важное свойство монотонного убывания по $p \in \mathcal{P}$ функции $p \lambda_{0}(p, \beta \omega)$ описано в лемме 12 из [6]. Введем обозначение $\|x\|_{p}:=\left(\int_{0}^{1}|x(t)|^{p} d t\right)^{1 / p}, p>0$. Как известно, при $p \geqslant 1$ функционал $\|x\|_{p}$ является нормой в банаховом пространстве $L^{p}=L^{p}[0,1]$ функций, $p$-я степень которых интегрируема на $[0,1]$.

Для фиксированного $p \in \mathcal{P}$ введем функцию

$$
\theta(s) \equiv \theta(s, p, \beta \omega):=\frac{1}{\beta \omega} \int_{\delta_{0}}^{s / b_{0}} \frac{d z}{\sqrt{\frac{1-\delta_{0}^{2}}{1-\delta_{0}^{p}}\left(1-z^{p}\right)-\left(1-z^{2}\right)}}, \quad h_{0} \leqslant s \leqslant b_{0} .
$$

Легко убедиться, что функция $\theta(s)$ монотонно возрастает по $s \in\left[h_{0}, b_{0}\right]$, при этом

$$
\theta\left(h_{0}\right)=0, \quad \theta\left(b_{0}\right)=\frac{J\left(\delta_{0}\right)}{\beta \omega}=\frac{1}{2},
$$

следовательно, однозначно определена обратная функция $\theta^{-1}$; положим

$$
x_{0}(t):=\theta^{-1}(t), \quad t \in[0,1 / 2] .
$$

Распространим функцию $x_{0}(t)$ на весь отрезок $[0,1]$ симметричным образом, положив

$$
x_{0}(t)=x_{0}(1-t), \quad t \in[1 / 2,1] .
$$

Очевидно, функция $x_{0}(t)$ является строго положительной, она монотонно возрастает при $t \in[0,1 / 2]$, причем $x_{0}(0)=h_{0}$ и $x_{0}(1 / 2)=b_{0}$. Таким образом, для всех значений $t \in[0,1]$ выполнены неравенства $0<h_{0} \leqslant x_{0}(t) \leqslant b_{0}$. Из результатов статьи [6] следует, что функция $x_{0}(t)$ дважды дифференцируема на отрезке $[0,1]$ и удовлетворяет нелинейной граничной задаче

$$
\begin{gathered}
x^{\prime \prime}(t)=\beta^{2} \omega^{2} x(t)-\lambda_{0} p x^{p-1}(t), \quad t \in[0,1], \\
x(0)=x(1)=h_{0}, \quad x^{\prime}(0)=x^{\prime}(1)=0,
\end{gathered}
$$

при этом $x_{0}^{\prime}(1 / 2)=0$ и $\left\|x_{0}\right\|_{p}=1$. Следуя работе [8], введем функции

$$
f_{0}(t)=\frac{x_{0}(t)}{\left\|x_{0}\right\|_{2}}, \quad f_{1}(t)=\frac{x_{0}^{\prime}(t)}{\left\|x_{0}^{\prime}\right\|_{2}}, \quad t \in[0,1] .
$$

Для формулировки основного результата нам понадобится следующее предложение. Ниже $\mu$ - спектральный параметр.

ПредЛОЖЕНИЕ 1. Пусть фиксировано $p \in \mathcal{P}$. Самосопряженная граничная задача на собственные числа для линейного дифберенциалъного уравнения второго порядка

$$
\begin{gathered}
-z^{\prime \prime}(t)+\beta^{2} \omega^{2} z(t)=\frac{p(p-1)}{\mu} x_{0}^{p-2}(t) z(t), \quad t \in[0,1] \\
z(0)=z(1), \quad z^{\prime}(0)=z^{\prime}(1),
\end{gathered}
$$

имеет последовательность собственных чисел $\left\{\mu_{n}\right\}_{n=0}^{\infty}$ следующего вида:

$$
\mu_{0}=\frac{p-1}{\lambda_{0}}>\mu_{1}=\frac{1}{\lambda_{0}}>\mu_{2}>\mu_{3} \geqslant \mu_{4}>\cdots>0,
$$


при этом $\sum_{n=0}^{\infty} \mu_{n}<\infty$. Максимальному собственному числу $\mu_{0}=(p-1) / \lambda_{0}$ соответствует $L^{2}$-нормированная положительная собственная функция $f_{0}(t)$, собственному числу $\mu_{1}=1 / \lambda_{0}$ соответствует $L^{2}$-нормированная собственная функиия $f_{1}(t)$ (см. формуль (1.12)); собственным числам $\mu_{k}, k=2,3, \ldots$, соответствуют $L^{2}$-нормированные собственные функции $f_{k}(t)$ и при этом система $\left\{f_{k}(t)\right\}_{k=0}^{\infty}$ образует ортонормированныц базис в $L^{2}$.

Предложение 1 доказано ниже в разделе 4. Основной результат настоящей работы состоит в следующем.

Теорема 1. Пусть заданы числа $m>0, \beta>0, \omega>0$ и $p \in \mathcal{P}$. Тогда для стаицонарного гауссовского прочесса Боголюбова $\xi(t), t \in[0, \beta]$, при $u \rightarrow \infty$ выполнено соотношение

$$
\mathbf{P}\left\{\int_{0}^{\beta}|\xi(t)|^{p} d t>u^{p}\right\}=c_{1} \exp \left\{-\frac{m \lambda_{0} p}{2 \beta^{(p+2) / p}} u^{2}\right\}(1+o(1)),
$$

при этом постоянная $c_{1}=c_{1}(p, \beta \omega)>0$ записъвается следующим образом:

$$
c_{1}=\frac{\left\|x_{0}^{\prime}\right\|_{2}^{2} \sqrt{2 \beta \omega \operatorname{sh}(\beta \omega / 2)}}{\pi \sqrt{p \lambda_{0} c_{2}}}\left[\prod_{k=2}^{\infty}\left(1-\lambda_{0} \mu_{k}\right)\right]^{-1 / 2},
$$

əде

$$
c_{2}=c_{2}(p, \beta \omega)=\int_{0}^{1} \int_{0}^{1} \operatorname{ch}\left(\beta \omega|t-s|-\frac{\beta \omega}{2}\right) x_{0}^{\prime}(t) x_{0}^{\prime}(s) d t d s>0 .
$$

ЗАМЕЧАНИЕ 2. Для $p \in \mathcal{P}$ положим

$$
C_{0}=C_{0}(p, \beta \omega)=2 \lambda_{0} b_{0}^{p}-\beta^{2} \omega^{2} b_{0}^{2}<0 .
$$

Отметим, что постоянные $c_{1}, c_{2}, C_{0}$, (как и величины $\left.\delta_{0}, h_{0}, b_{0}, \lambda_{0}\right)$ зависят только от $p$ и произведения $\beta \omega$, но не зависят от массы $m$. Несложно доказать, что справедливы равенства

$$
\begin{aligned}
& \left\|x_{0}\right\|_{2}^{2}=\int_{0}^{1} x_{0}^{2}(t) d t=2 \int_{h_{0}}^{b_{0}} \frac{s^{2} d s}{\sqrt{\beta^{2} \omega^{2} s^{2}-2 \lambda_{0} s^{p}+C_{0}}} \\
& \left\|x_{0}^{\prime}\right\|_{2}^{2}=\int_{0}^{1}\left[x_{0}^{\prime}(t)\right]^{2} d t=2 \int_{h_{0}}^{b_{0}} \sqrt{\beta^{2} \omega^{2} s^{2}-2 \lambda_{0} s^{p}+C_{0}} d s .
\end{aligned}
$$

\section{2. ДОКАЗАТЕЛЬСТВО ЛЕММЫ 1}

Первое утверждение леммы 1 (существование корня) легко получить из непрерывности функции $J_{p}(\delta)$ и соотношений $(1.4),(1.5)$. Доказательство второго утверждения леммы 1 (единственность корня) для значений $p \in \mathcal{P}$ (см. (1.6)) значительно сложнее, оно основано на свойстве монотонного убывания функции $J_{p}(\delta)$ на множестве $\delta \in(0,1]$. Это свойство мы доказываем, устанавливая, что при $p \in \mathcal{P}$ производная $J_{p}^{\prime}(\delta)<0$ для всех $\delta \in(0,1)$. Теорему 2 о существовании и виде производной $J_{p}^{\prime}(\delta)$ мы формулируем и доказываем ниже для общего случая $p>2$, это сделано с целью возможного обобщения п. 2 леммы 1 на другие значения параметра $p$. 
Для фиксированного числа $p>2$ введем следующую функцию:

$$
\phi(t) \equiv \phi_{p}(t):=\frac{1-t^{2}}{1-t^{p}}, \quad t \in[0,1), \quad \phi(1):=\lim _{t \uparrow 1} \phi(t)=\frac{2}{p} .
$$

ЛЕмма 2. Функция $\phi(t)$ строго монотонно убъвает на отрезке $t \in[0,1]$ от значения $\phi(0)=1$ до значения $\phi(1)=2 / p<1$. Для производной функции $\phi(t)$ выполнены соотношения

$$
\phi^{\prime}(t)=\frac{t\left[(2-p) t^{p}+p t^{p-2}-2\right]}{\left(1-t^{p}\right)^{2}}, \quad t \in[0,1],
$$

при этом $\phi^{\prime}(t)<0$ при всех $t \in(0,1] u$

$$
\phi^{\prime}(0)=0, \quad \phi^{\prime}(1):=\lim _{t \uparrow 1} \phi^{\prime}(t)=\frac{2-p}{p}<0 .
$$

Все утверждения леммы 2, кроме соотношения (2.3), доказаны в статье [6] (см. лемму 2). Первое равенство в формуле (2.3) вытекает непосредственно из соотношения (2.2), второе соотношение в формуле (2.3) легко получаем из $(2.2)$, применяя правило Лопиталя.

По аналогии с формулами (1.2), (1.3) для фиксированного числа $p>2$ введем следующую функцию от $\delta \in(0,1)$ :

$$
\Psi_{p}(\delta):=\int_{\delta}^{1} \frac{\left(t^{p}-\delta^{p}\right) d t}{\sqrt{1-t^{p}}[\phi(\delta)-\phi(t)]^{3 / 2}} .
$$

Несложно убедиться, что функция $\Psi_{p}(\delta)$ корректно определена и непрерывна. Отметим, что подынтегральная функция в (2.4) имеет интегрируемые особенности при $t=\delta$ и $t=1$.

Теорема 2. Для любого фиксированного числа $p>2$ функиия $J_{p}(\delta)$, заданная в (1.2), непрерывно дифберенцируема на множестве $\delta \in(0,1]$. Производная функиии $J_{p}(\delta)$ имеет вид

$$
J_{p}^{\prime}(\delta)=-\frac{Q_{p}(\delta)}{2 \delta\left(1-\delta^{p}\right)}, \quad 0<\delta \leqslant 1
$$

əəe

$$
Q_{p}(\delta):=2 p M_{p}(\delta)-(p-2) \phi(\delta) \Psi_{p}(\delta), \quad 0<\delta \leqslant 1 .
$$

При этом выполнены соотношения

$$
\begin{array}{cc}
J_{p}^{\prime}(\delta)=-\frac{1}{\delta}(1+o(1)), \quad \delta \downarrow 0, & J_{p}^{\prime}(1)=\lim _{\delta \uparrow 1} J_{p}^{\prime}(\delta)=0, \\
Q_{p}(0):=\lim _{\delta \downarrow 0} Q_{p}(\delta)=2, & Q_{p}(1)=\lim _{\delta \uparrow 1} Q_{p}(\delta)=0 .
\end{array}
$$

ДокАЗАТЕЛЬСтво теоремы 2 довольно длинное и здесь опущено.

Следующая лемма показывает, что локально в окрестности особой точки $\delta=1$ функция $J_{p}(\delta)$ строго монотонно убывает по $\delta$. 
ЛЕмма 3. При фиксированном $p>2$ справедливо асимптотическое разложение Тейлора

$$
J_{p}(\delta)=\frac{\pi}{\sqrt{p-2}}+\frac{\pi(p-1)(p+2)}{96 \sqrt{p-2}}(1-\delta)^{2}+C_{0}(1-\delta)^{3}+O(1-\delta)^{4}, \quad \delta \uparrow 1,
$$

где $C_{0}=C_{0}(p) \in \mathbb{R}$ - некоторая постоянная. Таким образом, при $p>2$ у функиии $J_{p}(\delta)$ в точке $\delta=1$ существуют левосторонние первая и вторая производные $J_{p}^{\prime}(1), J_{p}^{\prime \prime}(1)$ и при этом выполнены соотношения

$$
J_{p}^{\prime}(1):=\lim _{\delta \uparrow 1} J_{p}^{\prime}(\delta)=0, \quad J_{p}^{\prime \prime}(1)=\frac{\pi(p-1)(p+2)}{48 \sqrt{p-2}}>0 .
$$

ЗАМЕчАНИЕ 3. Из соотношения (2.8) следует, что при $p>2$ у функции $J_{p}(\delta)$ в точке $\delta=1$ существует левосторонняя третья производная $J_{p}^{\prime \prime \prime}(1)$. Отсюда следует, что в достаточно малой окрестности $U$ точки $\delta=1$ у функции $J_{p}(\delta)$ существует непрерывная вторая производная $J_{p}^{\prime \prime}(\delta)$ и при этом в силу последнего соотношения из (2.9) выполнено неравенство $J_{p}^{\prime \prime}(\delta)>0$ при $\delta \in U$. Таким образом, с учетом равенства $J_{p}^{\prime}(1)=0$ заключаем, что при $p>2$ в достаточно малой окрестности $U$ точки $\delta=1$ функция $J_{p}(\delta)$ выпукла вниз и строго монотонно убывает по $\delta$.

ДокАЗАТЕЛьство леммы 3 проводится по схеме доказательства леммы 5 в [6], оно достаточно длинное и здесь опущено.

Можно также доказать, что для фиксированного $p>2$ справедливо соотношение

$$
J_{p}(\delta)=-\ln \delta(1+o(1)), \quad \delta \downarrow 0 .
$$

Отметим, что формула (2.10) согласуется с формулой (2.7).

Из формулы (2.5) следует, что при фиксированном $p>2$ производная $J_{p}^{\prime}(\delta)<0$ для всех $\delta \in(0,1)$ тогда и только тогда, когда

$$
Q_{p}(\delta)>0 \quad \text { для всех } \delta \in(0,1) \text {. }
$$

Исследовать задачу о справедливости неравенства (2.11) для всех $p>2$, используя формулу (2.6), довольно сложно. Ниже мы дадим более удобную формулу для $Q_{p}(\delta)$ в случае, когда параметр $p$ принимает четные значения, большие двух. Введем функцию

$$
\Pi(n, \delta):=1+\delta^{2}+\delta^{4}+\cdots+\delta^{2 n-2}, \quad 0 \leqslant \delta \leqslant 1 .
$$

Тогда в силу формул $(2.1),(2.12)$ при $p=2 n, n=2,3,4, \ldots$, справедливы равенства

$$
\phi(\delta) \equiv \phi_{2 n}(\delta)=\frac{1}{\Pi(n, \delta)}=\frac{1}{1+\delta^{2}+\delta^{4}+\cdots+\delta^{2 n-2}}, \quad 0 \leqslant \delta \leqslant 1 .
$$

Для фиксированных целого $n \geqslant 2$ и $\delta \in(0,1)$ введем следующие функции:

$$
\begin{aligned}
G_{2 n}\left(t^{2}, \delta^{2}\right) & :=\sum_{k=0}^{n-2}\left[\sum_{j=0}^{n-2-k} \delta^{2 j}\right] t^{2 k}, \quad \delta \leqslant t \leqslant 1, \\
R_{\delta, 2 n}(t) & :=\phi_{2 n}(\delta)\left(1-t^{2 n}\right)-\left(1-t^{2}\right) \equiv \\
& \equiv \phi_{2 n}(\delta)\left(1-t^{2}\right)\left(t^{2}-\delta^{2}\right) G_{2 n}\left(t^{2}, \delta^{2}\right), \quad \delta \leqslant t \leqslant 1 .
\end{aligned}
$$


Второе соотношение в (2.12) справедливо в силу формул $(2.13),(2.14)$. Введем также функции

$$
L_{2 n}(\delta):=\int_{\delta}^{1} \frac{t^{2} d t}{\sqrt{R_{\delta, 2 n}(t)}}, \quad F_{2 n}(\delta):=\int_{\delta}^{1} \frac{d t}{\sqrt{R_{\delta, 2 n}(t)} G_{2 n}\left(t^{2}, \delta^{2}\right)}, \quad 0<\delta<1 .
$$

Справедливо следующее утверждение.

ПреДЛОЖЕНИЕ 2. Пусть фиксировано иелое $n \geqslant 2$. Тогда имеет место тождество

$$
\begin{aligned}
Q_{2 n}(\delta)= & 2\left[n+\left(\delta^{2}(n-1)-1\right) \Pi(n, \delta)\right] J_{2 n}(\delta)-2(n-3) \Pi(n, \delta) L_{2 n}(\delta)- \\
& -2(n-1) \Pi^{2}(n, \delta) F_{2 n}(\delta), \quad 0<\delta \leqslant 1 .
\end{aligned}
$$

ДокАЗАТЕЛЬСТво довольно длинное и здесь опущено. На основе формулы (2.16) доказывается п. 2 леммы 1.

\section{3. ТОЧНЫЕ АСИМПТОТИКИ БОЛЬШИХ УКЛОНЕНИЙ ДЛЯ ГАУССОВСКИХ МЕР: МНОГООБРАЗИЕ ТОЧЕК МИНИМУМА ФУНКЦИОНАЛА ДЕЙСТВИЯ}

Следуя статье [8], изложим один общий результат об асимптотиках больших уклонений гауссовских мер. При помощи этого результата мы докажем теорему 1.

Пусть $H$ - сепарабельное гильбертово пространство с нормой $\|\cdot\|$ и скалярным произведением $\langle\cdot, \cdot\rangle$. Пусть $P_{A}$ - гауссовская мера в гильбертовом пространстве $H$, имеющая нулевое среднее и инъективный ковариационный оператор $A$. В последующих формулах $\operatorname{Dom}(C)$ обозначает область определения оператора $C, \operatorname{Ran}(C)-$ область его значений, тождественные операторы в различных пространствах обозначаются одним и тем же символом $I$. Предположим, что выполнены следующие условия.

УСловиЕ В.1. Борелевское множество D замкнуто и имеет вид

$$
D=\{x \in H: Q(x) \geqslant 0\},
$$

где $Q(x)$ - непрерывная действительная функиия такая, что или $D$, или множество $H \backslash D$ представимо в виде конечного обгединения непересекающихся выпуклых множеств из $H$ и при этом $P_{A}\{x \in H:|Q(x)|<\infty\}=1$. Пусть $D$ имеет непустую внутренность и не содержит нулевого элемента из $H$.

УСловие В.2. Квадратичная форма $\left\langle x, A^{-1} x\right\rangle$ достигает своего минимума по $x \in D \cap \operatorname{Ran}(A)$, который равен $\sigma^{-2}>0$, на одномерном дважды дифберениируемом компактном многообразии $M$, лежащем на границе $\partial D$ множества $D$.

Таким образом,

$$
\left\langle m, A^{-1} m\right\rangle=\sigma^{-2}>0 \quad \text { для всех } m \in M .
$$

Обозначим через $n_{x} \in H$ единичный касательный вектор к кривой $M$ в точке $x$, через $\widehat{M}_{x}$ - одномерное аффинное пространство, натянутое на вектор $n_{x}$ и содержащее точку $x$, т. е. одномерное пространство, касательное к $M$ в точке $x$ (см. монографии [9], гл. 2, п. 2.3.5, и [10], гл. $3, \S 3)$. Тогда $M_{x}:=\widehat{M}_{x}-x$ представляет 
собой одномерное подпространство в $H$, содержащее точку 0. Как это принято, мы будем рассматривать единичные касательные векторы как свободные векторы, поэтому выходящий из точки 0 единичный вектор, на который натянуто подпространство $M_{x}$, мы также будем обозначать через $n_{x}$.

Имеет место разложение гильбертова пространства $H$ в прямую сумму подпространств $H=M_{x} \oplus N_{x}$, где $N_{x}$ - ортогональное дополнение подпространства $M_{x}$ коразмерности 1 [11]. Введем следующие два условия.

УСловие В.3. В пространстве Н существуют окрестность $U$ многообразия $M$ и множество $U_{0}$ такие, что $M \subset U_{0} \subset U$, функция $Q(x)$ трижды дифберенцируема по Фреше во всех точках $x \in U_{0}$ и $P_{A}\left(U \backslash U_{0}\right)=1$. Выполнено условие невырожденности: для всех $x \in M$ имеет место неравенство

$$
\left\langle y,\left[A^{-1}-\lambda Q^{\prime \prime}(x)\right] y\right\rangle>0, \quad 0 \neq y \in N_{x} \cap \operatorname{Dom}\left(A^{-1}\right) \cap \operatorname{Dom}\left(Q^{\prime \prime}(x)\right),
$$

где $\lambda=\left\langle x, A^{-1} x\right\rangle /\left\langle x, Q^{\prime}(x)\right\rangle>0$ - множитель Лагранжа.

УСловиЕ В.4. Для каждого $x \in M$ выполнено условие ортогональности

$$
\left\langle x, n_{x}\right\rangle=0 .
$$

Для каждого фиксированного $x \in M$ выберем с учетом (3.1) некоторый ортонормированный базис $\left\{e_{k}^{x}\right\}_{k=0}^{\infty}$ в гильбертовом пространстве $H$ так, чтобы первые два элемента базиса имели следующий вид:

$$
e_{0}^{x}=n_{x}, \quad e_{1}^{x}=\frac{x}{\|x\|} .
$$

Пусть $\Lambda(x)$ - линейный оператор, имеющий в базисе $\left\{e_{k}^{x}\right\}_{k=0}^{\infty}$ матричное представление

$$
\Lambda(x)=\left[\begin{array}{ccc}
0 & 0 & A^{-1} x \\
0 & 0 & n_{x} \\
A n_{x} & x & I-\lambda A Q^{\prime \prime}(x)
\end{array}\right]
$$

Формула (3.3) понимается в следующем координатном смысле. Элементы $A^{-1} x \in H$ и $n_{x}=e_{0}^{x}=(1,0,0, \ldots) \in H$ записываются в виде вектор-строк своих координат, элементы $A n_{x} \in H$ и $x=\|x\| e_{1}^{x}=(0,\|x\|, 0,0, \ldots) \in H-$ в виде вектор-столбцов, а оператор (вообще говоря, недиагональный) $I-\lambda A Q^{\prime \prime}(x): H \rightarrow H-$ в матричном виде в базисе $\left\{e_{k}^{x}\right\}_{k=0}^{\infty}$. Матрица $\Lambda(x)$ состоит из этих блоков и двумерной нулевой матрицы, стоящей в верхнем левом углу.

Условие В.5. Для каждого $x \in M$ выполнено условие $\operatorname{det} \Lambda(x) \neq 0$.

Положим

$$
c(x)=|\operatorname{det} \Lambda(x)|^{-1 / 2}, \quad x \in M .
$$

Теорема 3. Пусть $P_{A}$ - гауссовская мера в гильбертовом пространстве $H$, имеющая нулевое среднее и инбективный ковариационный оператор А. Пусть выполнены условия В.1-В.5. Тогда при $u \rightarrow \infty$ справедливо асимптотическое соотношение

$$
P_{A}(u D)=\exp \left\{-\frac{u^{2}}{2 \sigma^{2}}\right\} \frac{1}{2 \pi} \int_{M} c(x) d V_{M}(x)(1+o(1)),
$$

где $c(x)$ задано в (3.4), $d V_{M}$ - элемент длинь на кривой $M$. 
Утверждение теоремы 3 вытекает непосредственно из теоремы 1 и замечания 1 статьи [8], где рассмотрен общий случай гауссовской меры в банаховом пространстве.

Замечание 4. Довольно часто оператор $A$ и множества $D$ и $M$ обладают некоторыми свойствами симметрии (подробное определение имеется в работах [12], [13], см. также статью [14]). В такой ситуации функция $c(x)$ принимает одно и то же значение $c$ для всех точек $x \in M$ и $\int_{M} c(x) d V_{M}(x)=c \cdot \operatorname{mes}(M)$, где $\operatorname{mes}(M)$ - длина кривой $M$. Именно такой случай возникает при доказательстве теоремы 1.

ЗАмечАниЕ 5. Элементы базиса $e_{k}^{x}, k=2,3, \ldots$, целесообразно выбирать таким образом, чтобы матричное представление оператора $I-\lambda A Q^{\prime \prime}(x)$ в базисе $\left\{e_{k}^{x}\right\}_{k=0}^{\infty}$ имело наиболее простой вид.

\section{4. ДОКАЗАТЕЛЬСТВО ТЕОРЕМЫ 1}

В силу масштабного свойства процесса Боголюбова (см. лемму 6 в [6]), для любых значений $p>0$ и $u>0$ выполнено равенство

$$
\mathbf{P}\left\{\int_{0}^{\beta}|\xi(t)|^{p} d t>u^{p}\right\}=\mathbf{P}\left\{\int_{0}^{1}|\eta(s)|^{p} d s>v^{p}\right\}, \quad v=\frac{u \sqrt{m}}{\beta^{(2+p) / 2 p}},
$$

где

$$
\eta(t)=\frac{\sqrt{m}}{\sqrt{\beta}} \xi(\beta t), \quad t \in[0,1],
$$

- гауссовский процесс Боголюбова на отрезке $[0,1]$ с параметрами $\left(1,1, \omega_{1}\right), \omega_{1}=\beta \omega$. Таким образом, в силу формулы (4.1) нам достаточно доказать вариант теоремы 1 с большим параметром $v \rightarrow \infty$ для процесса Боголюбова $\eta(t)$.

Доказательство теоремы 1 проведем при помощи теоремы 3. Как и в статье [6], возьмем в качестве гильбертова пространства $H$ обычное гильбертово пространство $L^{2} \equiv L^{2}[0,1]$ действительных измеримых функций на $[0,1]$, квадрат которых интегрируем, со скалярным произведением и нормой,

$$
\langle x, y\rangle=\int_{0}^{1} x(t) y(t) d t, \quad\|x\|_{2}=\left(\int_{0}^{1} x^{2}(t) d t\right)^{1 / 2}, \quad x, y \in L^{2}[0,1] .
$$

Обозначим через $\mu_{\eta}$ распределение процесса $\eta(t)$ в пространстве $C^{0}[0,1]$.

Мы будем рассматривать гауссовскую меру $\mu_{\eta}$, полагая ее заданной в пространстве $L^{2}[0,1]$, при этом будем иметь в виду, что эта мера сосредоточена на $C^{0}[0,1]$. Как известно, в этом случае $\mu_{\eta}$ имеет нулевое среднее и ковариационный оператор $A_{\eta}: L^{2} \rightarrow L^{2}$ интегрального типа:

$$
A_{\eta} x(t)=\int_{0}^{1}[\mathbf{E} \eta(t) \eta(s)] x(s) d s=\frac{1}{2 \omega_{1} \operatorname{sh}\left(\omega_{1} / 2\right)} \int_{0}^{1} \operatorname{ch}\left(\omega_{1}|t-s|-\frac{\omega_{1}}{2}\right) x(s) d s .
$$

Пусть $C^{2}[0,1]$ - множество дважды дифференцируемых на $[0,1]$ функций $x(t)$. Как указано в лемме 7 из работы [6], область значений ковариационного оператора $A_{\eta}$ имеет вид

$$
\operatorname{Ran}\left(A_{\eta}\right)=\left\{x \in C^{2}[0,1]: x(0)=x(1), x^{\prime}(0)=x^{\prime}(1)\right\} .
$$


Несложно показать, что оператор $A_{\eta}$ является инъективным и обратный оператор задается формулой

$$
\left[A_{\eta}^{-1} y\right](t)=-y^{\prime \prime}(t)+\omega_{1}^{2} y(t), \quad y \in \operatorname{Ran}\left(A_{\eta}\right), \quad t \in[0,1] .
$$

Учитывая (4.1), возьмем в качестве функционала $Q$, участвующего в формулировке теоремы 3 , следующий функционал:

$$
G(x):=\int_{0}^{1}|x(t)|^{p} d t-1, \quad x \in L^{2}, \quad p>2 .
$$

Очевидно, $\mu_{\eta}\left\{x \in L^{2}:|G(x)|<\infty\right\}=1$.

ЛЕмма 4. При р > 2 функционал $G$ является выпуклым функционалом, дважды дифференцируемым по Фреше в каждой точке $x \in C^{0}[0,1]$, с производными

$$
\begin{aligned}
G^{\prime}(x) & =p|x(t)|^{p-1} \operatorname{sgn} x(t), \\
G^{\prime \prime}(x) & =p(p-1)|x(t)|^{p-2},
\end{aligned}
$$

которые задают ограниченные линейные операторы. При $p>3$ функционал $G$ трижды дифберенцируемым по Фреше в каждой точке $x \in C^{0}[0,1]$, третъя производная задается бормулой

$$
G^{\prime \prime \prime}(x)[h, g, v]=\int_{0}^{1} p(p-1)(p-2)|x(t)|^{p-3} \operatorname{sgn} x(t) h(t) g(t) v(t) d t, \quad h, g, v \in L^{2} .
$$

Положим

$$
D=\left\{x \in L^{2}: G(x) \geqslant 0\right\} .
$$

В силу леммы 4 множество $L^{2} \backslash D$ является выпуклым при $p>2$. Очевидно, что множество $D$ из (4.8) имеет непустую внутренность и не содержит нуль. Таким образом, множество (4.8) удовлетворяет условию В.1 с $H=L^{2}[0,1]$.

Обратимся к условию В.2, полагая далее, что $p \in \mathcal{P}$. Несложно убедиться, что в силу формул $(1.11),(4.3)$ функция $x_{0}(t)$, заданная в $(1.9),(1.10)$, удовлетворяет на отрезке $[0,1]$ периодическим граничным условиям и $x_{0}(t) \in \operatorname{Ran}\left(A_{\eta}\right)$.

Продолжим периодически функцию $x_{0}(t)$ на всю ось $\mathbb{R}$, полученную периодическую функцию с периодом единица обозначим через $\widehat{x}_{0}(t), t \in \mathbb{R}$. Для числа $0 \leqslant \kappa \leqslant 1$ определим функцию

$$
x_{\kappa}(t):=\widehat{x}_{0}(t+\kappa), \quad t \in[0,1] .
$$

Очевидно, $x_{0}(t) \equiv x_{1}(t)$. Кроме того, в силу определения функции (4.9) и ее периодичности при всех $0 \leqslant \kappa \leqslant 1$ имеют место соотношения $x_{\kappa}(t) \in \operatorname{Ran}\left(A_{\eta}\right),\left\|x_{\kappa}\right\|_{p}=1$. Введем множество $\mathcal{M}_{0}$ функций, заданных на $[0,1]$, как

$$
\mathcal{M}_{0}:=\left\{x_{\kappa}(t): 0 \leqslant \kappa \leqslant 1\right\} .
$$

Отметим, что в (4.10) элементы $x_{0}$ и $x_{1}$ отождествлены, следовательно, многообразие $\mathcal{M}_{0}$ является замкнутой компактной кривой, которая принадлежит множеству $\operatorname{Ran}\left(A_{\eta}\right)$. Зададим также множество, центрально-симметричное множеству $\mathcal{M}_{0}$ :

$$
\widetilde{\mathcal{M}}_{0}:=-\mathcal{M}_{0}=\left\{-x(t): x(t) \in \mathcal{M}_{0}\right\} .
$$

Напомним, что число $\lambda_{0}>0$ задано в формуле (1.8). 
Лемма 5. Пусть фиксировано число $p \in \mathcal{P}$. Тогда минимум в экстремальной задаче

$$
\left\langle x, A_{\eta}^{-1} x\right\rangle \rightarrow \inf , \quad x \in D \cap \operatorname{Dom}\left(A_{\eta}^{-1}\right),
$$

равен $\lambda_{0} p$ и достигается на одномерном компактном двусвязном многообразии

$$
\mathcal{M}:=\mathcal{M}_{0} \cup \widetilde{\mathcal{M}}_{0} \subset \partial D \cap \operatorname{Ran}\left(A_{\eta}\right) .
$$

ДокАЗАТЕЛЬСТво проводится при помощи метода, использованного при доказательстве леммы 10 в [6], с учетом п. 2 леммы 1 и замечания 1 из раздела 1.

Охарактеризуем более точно замкнутые многообразия (4.10), (4.11), заданные для значений $p \in \mathcal{P}$. Напомним, что по определению все функции, принадлежащие множеству $\mathcal{M}_{0}$, являются строго положительными.

Лемма 6. 1. Имеют место равенства

$$
\operatorname{mes}\left(\mathcal{M}_{0}\right)=\operatorname{mes}\left(\widetilde{\mathcal{M}}_{0}\right)=\left\|x_{0}^{\prime}\right\|_{2}
$$

где $\operatorname{mes}\left(\mathcal{M}_{0}\right)$ - лебегова длина замкнутой компактной кривой $\mathcal{M}_{0}$.

2. Для любого $\kappa \in[0,1]$ справедливы следующие равенства для норм функиий из многообразия $\mathcal{M}_{0}$ и их производных:

$$
\left\|x_{\kappa}\right\|_{2}=\left\|x_{0}\right\|_{2}, \quad\left\|x_{\kappa}^{\prime}\right\|_{2}=\left\|x_{0}^{\prime}\right\|_{2} .
$$

3. Основная экстремаль $x_{0}(t)$ не обладает симметрией на отрезке $[0,1 / 2]$ относительно точки $t=1 / 4$, т.е. нельзя утверждать, что

$$
x_{0}(t)=h_{0}+b_{0}-x_{0}\left(\frac{1}{2}-t\right) \quad \text { для всех } t \in\left[0, \frac{1}{2}\right] .
$$

4. Многообразие $\mathcal{M}_{0}$, заданное в (4.10) и рассматриваемое в гилъбертовом пространстве $L^{2}$, представляет собой дважды дифберениируемую замкнутую компактную одномерную кривую, лежащую на пересечении конуса положительных бункиий $\mathcal{K}$ и сферь радиуса $\left\|x_{0}\right\|_{2}$ в $L^{2}$. Кривая $\mathcal{M}_{0}$ не является окружностью.

ДокАЗАТЕЛЬство вытекает непосредственно из утверждений работы [6] с учетом п. 2 леммы 1 из раздела 1 настоящей статьи (см., в частности, формулу (5.26) в работе [6]).

В силу лемм 5,6 для гауссовской меры $\mu_{\eta}$ и множества $D$ из (4.8) при $p \in \mathcal{P}$ выполнено условие В.2 с многообразием $\mathcal{M}$, заданным в $(4.12)$, и $\sigma^{-2}=\lambda_{0} p$. Учитывая лемму 4 и предложение 1 , доказанное ниже, несложно показать, что для выпуклого функционала $G$ из (4.5) и многообразия $\mathcal{M}$ из (4.12) выполнено условие В.3 с $\lambda=\lambda_{0}$.

Покажем, что для гладкого многообразия $\mathcal{M}$ выполнено условие В.4. Согласно своему определению (см. [10], гл. $3, \S 3$, п. 2) касательный вектор к кривой (4.10) в точке $x_{0}$ имеет вид $\left.\frac{d x_{\kappa}}{d \kappa}\right|_{\kappa=0}$. Используя тот факт, что $\kappa$ в формулах $(4.9),(4.10)$ является параметром сдвига, находим $\left.\frac{d x_{\kappa}}{d \kappa}\right|_{\kappa=0}=x_{0}^{\prime}(t), t \in[0,1]$. Таким образом, единичный касательный вектор к кривой $(4.10)$ в точке $x_{0}$ имеет вид (cp. с (1.12))

$$
n_{x_{0}}(t) \equiv n_{0}(t)=\frac{x_{0}^{\prime}(t)}{\left\|x_{0}^{\prime}\right\|_{2}}, \quad t \in[0,1] .
$$


Отсюда получаем

$$
\left\langle x_{0}, n_{0}\right\rangle=\frac{1}{\left\|x_{0}^{\prime}\right\|_{2}} \int_{0}^{1} x_{0}(t) x_{0}^{\prime}(t)(t) d t=\frac{1}{2\left\|x_{0}^{\prime}\right\|_{2}} \int_{0}^{1} d x_{0}^{2}(t)=0,
$$

так как $x_{0}(0)=x_{0}(1)$ согласно (1.10). Аналогично доказывается равенство (3.1) для всех $x \in \mathcal{M}_{0}$ и всех $x \in \widetilde{\mathcal{M}}_{0}$. Итак, для многообразия $\mathcal{M}$ выполнено условие В.4.

Отметим также, что в силу формул (1.10), (1.11) выполнены равенства

$$
x_{0}^{\prime}(0)=x_{0}^{\prime}(1)=0, \quad x_{0}^{\prime \prime}(0)=x_{0}^{\prime \prime}(1) .
$$

Из этих равенств и из соотношений $(1.12),(4.3),(4.13)$ следует, что $x_{0}^{\prime} \in \operatorname{Ran}\left(A_{\eta}\right)$ и $n_{0} \equiv f_{1} \in \operatorname{Ran}\left(A_{\eta}\right)$.

ДоКАЗАТЕЛЬСТВо ПРЕДЛОЖЕНИЯ 1 . В силу леммы 4 оператор $G^{\prime \prime}\left(x_{0}\right): L^{2} \rightarrow L^{2}$ является ограниченным; напомним, что ковариационный оператор $A_{\eta}: L^{2} \rightarrow L^{2}$ является ядерным. Поскольку множество ядерных операторов, действующих на всем классе банаховых пространств, является операторным идеалом [15], результирующий оператор $A_{\eta} G^{\prime \prime}\left(x_{0}\right)$ представляет собой ядерный оператор.

В силу соотношений $(4.3),(4.4)$ задача на собственные числа

$$
A_{\eta} G^{\prime \prime}\left(x_{0}\right) z=\mu z, \quad z \in \operatorname{Ran}\left(A_{\eta}\right) \subset L^{2},
$$

эквивалентна самосопряженной граничной задаче (1.13) на собственные числа. Из формулы (1.13) вытекают следующие соотношения для $L^{2}$-нормированных собственных функций $z(t)$ :

$$
\frac{p(p-1)}{\mu} \int_{0}^{1} x_{0}^{p-2}(t) z^{2}(t) d t=-\int_{0}^{1} z^{\prime \prime}(t) z(t) d t+\beta^{2} \omega^{2} \int_{0}^{1} z^{2}(t) d t>0
$$

так как в силу граничных условий задачи (1.13)

$$
-\int_{0}^{1} z^{\prime \prime}(t) z(t) d t=-\int_{0}^{1} z(t) d z^{\prime}(t)=\int_{0}^{1}\left[z^{\prime}(t)\right]^{2} d t \geqslant 0 .
$$

В нашем случае $p>p_{0}>2$ и $x_{0}(t)>0$ при $t \in[0,1]$, кроме того $\int_{0}^{1} x_{0}^{p-2}(t) z^{2}(t) d t>0$ при $z \neq 0$, тогда из формулы (4.17) вытекает, что $A_{\eta} G^{\prime \prime}\left(x_{0}\right)$ является положительным ядерным оператором и имеет последовательность положительных собственных чисел $\left\{\mu_{n}\right\}_{n=0}^{\infty}$ такую, что $\sum_{n=0}^{\infty} \mu_{n}<\infty$. Этой последовательности собственных чисел соответствует система $L^{2}$-нормированных собственных функций $\left\{f_{k}(t)\right\}_{k=0}^{\infty}$, которая образует ортонормированный базис в $L^{2}$. Докажем, что $f_{0}$ и $f_{1}$ имеют вид (1.12).

Согласно формуле (1.11) для минимизирующей функции $x_{0}(t)$ выполнены следующие соотношения:

$$
\begin{gathered}
x_{0}^{\prime \prime}(t)=\beta^{2} \omega^{2} x_{0}(t)-\lambda_{0} p x_{0}^{p-1}(t), \quad t \in[0,1], \\
x_{0}(0)=x_{0}(1), \quad x_{0}^{\prime}(0)=x_{0}^{\prime}(1)=0 .
\end{gathered}
$$

Используя формулу (4.18), несложно убедиться, что $\mu_{0}=(p-1) / \lambda_{0}$ является собственным числом задачи (1.13) с соответствующей $L^{2}$-нормированной положительной собственной функцией $f_{0}(t)$, которая задана в (1.12). Дифференцируя почленно 
уравнение из формулы (4.18) и учитывая (4.15), несложно показать, что $\mu_{1}=1 / \lambda_{0}$ является собственным числом задачи (1.13) с соответствующей $L^{2}$-нормированной собственной функцией $f_{1}(t)$, которая также задана в (1.12). В силу осцилляционной теоремы Камке (см. монографии [16], ч. I, п. 9.2, и [17], гл. 8, теорема 3.1)

$$
\mu_{0}=\frac{p-1}{\lambda_{0}}>\mu_{1}=\frac{1}{\lambda_{0}} \geqslant \mu_{2}>\mu_{3} \geqslant \mu_{4}>\cdots>0 .
$$

Покажем, что в (4.19) выполнено строгое неравенство $\mu_{1}>\mu_{2}$ и, следовательно, справедлива формула (1.14). Предположим противное, а именно, что

$$
\mu_{1}=\mu_{2}=\frac{1}{\lambda_{0}} .
$$

Отсюда следует, что ортогональные собственные функции $f_{1}(t)=x_{0}^{\prime}(t) /\left\|x_{0}^{\prime}\right\|_{2}$ и $f_{2}(t)$ удовлетворяют одной и той же граничной задаче

$$
\begin{aligned}
-z^{\prime \prime}(t)+\beta^{2} \omega^{2} z(t) & =\lambda_{0} p(p-1) x_{0}^{p-2}(t) z(t), \quad t \in[0,1], \\
z(0) & =z(1), \quad z^{\prime}(0)=z^{\prime}(1) .
\end{aligned}
$$

При этом выполнено соотношение ортогональности $\left\langle f_{0}, f_{2}\right\rangle=0$, т. е.

$$
\left\langle x_{0}, f_{2}\right\rangle=0 .
$$

Докажем, что выполнено также соотношение

$$
\left\langle x_{0}^{\prime \prime}, f_{2}\right\rangle=0 .
$$

В самом деле, полагая в $(4.21) z(t)=f_{2}(t)$, получаем с учетом (4.22) равенство

$$
-\left\langle f_{2}^{\prime \prime}, x_{0}\right\rangle=\lambda_{0} p(p-1) \int_{0}^{1} x_{0}^{p-1}(t) f_{2}(t) d t .
$$

С другой стороны, в силу формул (4.18), (4.22) справедливо равенство

$$
-\left\langle x_{0}^{\prime \prime}, f_{2}\right\rangle=\lambda_{0} p \int_{0}^{1} x_{0}^{p-1}(t) f_{2}(t) d t .
$$

Интегрируя по частям и используя периодические граничные условия из формул (4.18), (4.21), получаем, что

$$
\left\langle f_{2}^{\prime \prime}, x_{0}\right\rangle=\left\langle x_{0}^{\prime \prime}, f_{2}\right\rangle .
$$

Из формул (4.24)-(4.26) вытекает соотношение (4.23). Рассуждая так же, как при выводе формулы (4.26), несложно показать, что с учетом (4.23) справедливы равенства

$$
\int_{0}^{1} x_{0}^{\prime}(t) f_{2}^{\prime}(t) d t=-\int_{0}^{1} x_{0}^{\prime \prime}(t) f_{2}(t) d t=0 .
$$

Далее, две линейно независимые функции $x_{0}^{\prime}(t)$ и $f_{2}(t)$ удовлетворяют дифференциальному уравнению из формулы (4.21). Как известно (см., например, книгу [18], гл. $5, \S 1)$, для вронскиана этой пары выполнены соотношения

$$
\mathcal{W}\left(x_{0}^{\prime}, f_{2}\right):=x_{0}^{\prime}(t) f_{2}^{\prime}(t)-x_{0}^{\prime \prime}(t) f_{2}(t)=C, \quad t \in[0,1],
$$


где $C$ - некоторая ненулевая постоянная. Интегрируя почленно последнее равенство в (4.28) и используя (4.27), получаем соотношение $C=0$. Полученное противоречие показывает, что предположение (4.20) неверно и выполнено неравенство $\mu_{1}>\mu_{2}$. Таким образом, с учетом эквивалентности задач (1.13) и (4.16) мы доказали все утверждения предложения 1.

Итак, для значений $p \in \mathcal{P}$ мы применяем к гауссовскому процессу $\eta(t), t \in[0,1]$, теорему 3 , где нужно положить

$$
\begin{gathered}
H=L^{2}[0,1], \quad Q(x)=\int_{0}^{1}|x(t)|^{p} d t-1, \quad x \in L^{2}, \\
A=A_{\eta}, \quad P_{A}=P_{A_{\eta}}=\mu_{\eta}, \quad M=\mathcal{M}=\mathcal{M}_{0} \cup \widetilde{\mathcal{M}}_{0}, \quad \sigma^{-2}=\lambda_{0} p .
\end{gathered}
$$

Обратимся к последнему условию В.5. Учитывая свойство периодичности в определении многообразий $\mathcal{M}_{0}, \widetilde{\mathcal{M}}_{0}$, лемму 6 и формулы $(3.3),(3.4)$, можно показать, что в условиях теоремы 1 выполнены равенства

$$
c(x)=c\left(x_{0}\right)=\left|\operatorname{det} \Lambda\left(x_{0}\right)\right|^{-1 / 2}, \quad x \in \mathcal{M} .
$$

Вычислим $\left|\operatorname{det} \Lambda\left(x_{0}\right)\right|$. Используя предложение 1 , для точки $x_{0} \in \mathcal{M}$ возьмем в качестве ортонормированного базиса $\left\{e_{k}\right\}_{k=0}^{\infty}$ в $L^{2}$ систему функций $\left\{f_{k}\right\}_{k=0}^{\infty}$ из предложения 1 , при этом в соответствии с (3.2) положим

$$
e_{0}=f_{1}=n_{0}=\frac{x_{0}^{\prime}}{\left\|x_{0}^{\prime}\right\|_{2}}, \quad e_{1}=f_{0}=\frac{x_{0}}{\left\|x_{0}\right\|_{2}}, \quad e_{k}=f_{k}, \quad k=2,3, \ldots
$$

Обозначим координаты векторов $A_{\eta} n_{0}, A_{\eta}^{-1} x_{0}$ в базисе $\left\{e_{k}\right\}_{k=0}^{\infty}$ следующим образом:

$$
a_{k}:=\left\langle A_{\eta} n_{0}, e_{k}\right\rangle, \quad b_{k}:=\left\langle A_{\eta}^{-1} x_{0}, e_{k}\right\rangle, \quad k=0,1,2, \ldots
$$

Тогда матрица $\Lambda\left(x_{0}\right)$ из формулы (3.3) имеет следующий вид

$$
\Lambda\left(x_{0}\right)=\left[\begin{array}{ccccccc}
0 & 0 & b_{0} & b_{1} & b_{2} & b_{3} & \ldots \\
0 & 0 & 1 & 0 & 0 & 0 & \ldots \\
a_{0} & 0 & 0 & 0 & 0 & 0 & \ldots \\
a_{1} & \left\|x_{0}\right\|_{2} & 0 & 2-p & 0 & 0 & \ldots \\
a_{2} & 0 & 0 & 0 & 1-\lambda_{0} \mu_{2} & 0 & \ldots \\
a_{3} & 0 & 0 & 0 & 0 & 1-\lambda_{0} \mu_{3} & \ddots \\
\vdots & \vdots & \vdots & \vdots & \vdots & \ddots & \ddots
\end{array}\right] .
$$

Разлагая определитель $k$-мерной матрицы, аппроксимирующей матрицу (4.33), сначала по второй строке и второму столбцу, затем опять по второй строке и второму столбцу, получаем в результате диагональную матрицу, определитель которой легко считается. Таким образом,

$$
\left|\operatorname{det} \Lambda\left(x_{0}\right)\right|=a_{0} b_{1}\left\|x_{0}\right\|_{2} \prod_{k=2}^{\infty}\left(1-\lambda_{0} \mu_{k}\right) .
$$


Согласно равенствам (4.31), (4.32), (4.34) и лемме 5 справедливы соотношения

$$
\begin{gathered}
a_{0}=\left\langle A_{\eta} n_{0}, n_{0}\right\rangle, \quad b_{1}=\frac{\left\langle A_{\eta}^{-1} x_{0}, x_{0}\right\rangle}{\left\|x_{0}\right\|_{2}}=\frac{\lambda_{0} p}{\left\|x_{0}\right\|_{2}}, \\
\left|\operatorname{det} \Lambda\left(x_{0}\right)\right|=\lambda_{0} p\left\langle A_{\eta} n_{0}, n_{0}\right\rangle \prod_{k=2}^{\infty}\left(1-\lambda_{0} \mu_{k}\right) .
\end{gathered}
$$

Учитывая формулы (4.13), (4.30), (4.35), получаем равенства

$$
\begin{aligned}
\frac{1}{2 \pi} \int_{\mathcal{M}} c(x) d V_{\mathcal{M}}(x) & =\frac{c\left(x_{0}\right)}{\pi} \operatorname{mes}\left(\mathcal{M}_{0}\right)= \\
& =\frac{\left\|x_{0}^{\prime}\right\|_{2}}{\pi \sqrt{\lambda_{0} p\left\langle A_{\eta} n_{0}, n_{0}\right\rangle}}\left[\prod_{k=2}^{\infty}\left(1-\lambda_{0} \mu_{k}\right)\right]^{-1 / 2} .
\end{aligned}
$$

Далее, в силу формулы (4.2) выполнено равенство

$$
\left\langle A_{\eta} n_{0}, n_{0}\right\rangle=\frac{1}{2 \beta \omega \operatorname{sh}(\beta \omega / 2)\left\|x_{0}^{\prime}\right\|_{2}^{2}} \int_{0}^{1} \int_{0}^{1} \operatorname{ch}\left(\beta \omega|t-s|-\frac{\beta \omega}{2}\right) x_{0}^{\prime}(t) x_{0}^{\prime}(s) d t d s .
$$

Из формул (4.36), (4.37) следует, что

$$
\frac{1}{2 \pi} \int_{\mathcal{M}} c(x) d V_{\mathcal{M}}(x)=c_{1},
$$

где постоянная $c_{1}$ задана в (1.16), (1.17). Теперь, учитывая формулы (3.5), (4.29), (4.38), мы убеждаемся, что при $v \rightarrow \infty$ справедливо соотношение

$$
\mathbf{P}\left\{\int_{0}^{1}|\eta(s)|^{p} d s>v^{p}\right\}=\exp \left\{-\frac{\lambda_{0} p}{2} v^{2}\right\} c_{1}(1+o(1)) .
$$

Из формул (4.1), (4.39) вытекает соотношение (1.15) теоремы 1. Теорема 1 доказана.

Благодарности. Автор выражает благодарность рецензенту за полезные замечания.

\section{Список литературы}

[1] Д. П. Санкович, "Гауссовы функциональные интегралы и гиббсовские равновесные средние", ТМФ, 119:2 (1999), 345-352.

[2] Д. П. Санкович, "О некоторых свойствах функциональных интегралов по мере Боголюбова", ТМФ, 126:1 (2001), 149-163.

[3] Д. П. Санкович, "Метрические свойства боголюбовских траекторий в теории статистического равновесия", ТМФ, 127:1 (2001), 125-142.

[4] Н.Н. Боголюбов, "О представлении функций Грина-Швингера при помощи функциональных интегралов", Докл. АН СССР, 99:2 (1954), 225-226; Собрание научных трудов в 12 томах, т. 9: Квантовая теория поля, Наука, М., 2007, 245-247.

[5] Г. Крамер, М. Лидбеттер, Стачионарные случайные прочессы. Свойства выборочных бункиий и их приложения, Мир, М., 1969.

[6] В. Р. Фаталов, “Точные асимптотики типа Лапласа для гауссовской меры Боголюбова”, TMФ, 168:2 (2011), 299-340. 
[7] В.Р. Фаталов, "Ряды теории возмущения в квантовой механике: фазовые переходы и точные асимптотики для коэффициентов разложения", ТМФ, 174:3 (2013), 416-443.

[8] В.Р. Фаталов, "О методе Лапласа для гауссовских мер в банаховом пространстве", Теория вероятн. и ее примен., 58:2 (2013), 325-354.

[9] В. М. Алексеев, В.М. Тихомиров, С. В. Фомин, Оптимальное управление, Наука, М., 1979.

[10] А. С. Мищенко, А. Т. Фоменко, Курс дифференциальной геометрии и топологии, Из-во Моск. ун-та, М., 1980.

[11] С. Г. Крейн (ред.), Функииональный анализ, Наука, М., 1972.

[12] R.S. Ellis, J.S. Rosen, "Asymptotic analysis of Gaussian integrals I: Isolated minimum points", Trans. Amer. Math. Soc., 273:2 (1982), 447-481.

[13] R. S. Ellis, J. S. Rosen, "Asymptotic analysis of Gaussian integrals II: Manifold of minimum points", Commun. Math. Phys., 82:2 (1981), 153-181.

[14] В. И. Питербарг, В. Р. Фаталов, "Метод Лапласа для вероятностных мер в банаховых пространствах", УМН, 50:6(306) (1995), 57-150.

[15] А. Пич, Операторные идеаль, Мир, М., 1982.

[16] Э. Камке, Справочник по обыкновенным дифференииальным уравнениям, Физматгиз, M., 1965.

[17] Э.А. Коддингтон, Н. Левинсон, Теория обыкновенных дифференииальных уравнений, УРСС, М., 2007.

[18] Ф. Олвер, Асимптотики и специальные функиии, Наука, М., 1990.

Поступила в редакцию 9.02.2016, после доработки 29.04.2016 